\title{
KULEUVEN
}

DEPARTMENT OF ECONOMICS

\section{Measuring cumulative deprivation and affluence based on the diagonal dependence diagram}

Koen DECANCQ

FACULTY OF ECONOMICS AND BUSINESS

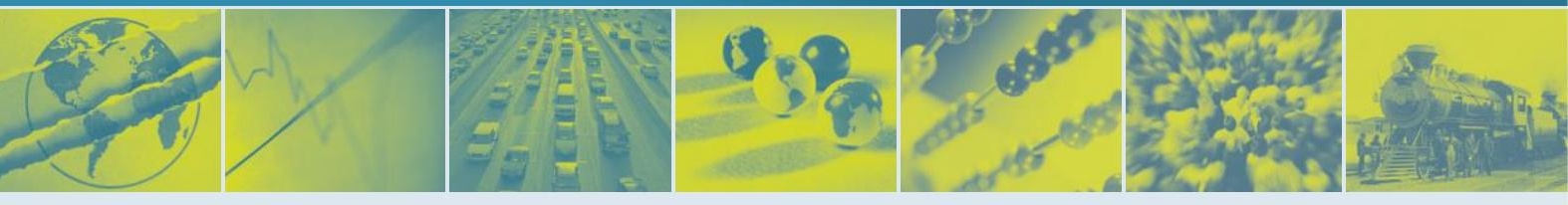

DISCUSSION PAPER SERIES DPS20.02

MARCH 2020 


\title{
Measuring cumulative deprivation and affluence based on the diagonal dependence diagram*
}

\author{
Koen Decancq ${ }^{\dagger}$
}

March 20, 2020

\begin{abstract}
In this paper, I introduce the diagonal dependence diagram to chart dependence around the main diagonal of a multivariate distribution. This diagonal dependence diagram is a useful tool to quantify phenomena such as cumulative deprivation and affluence. A society is said to exhibit more cumulative deprivation when more persons occupy bottom positions in all dimensions of well-being. Analogously, there is more cumulative affluence in a society when more persons occupy top positions in all dimensions. The diagonal dependence diagram consists of two curves which are obtained by taking the diagonal section of the underlying copula and survival functions, respectively. I show the elementary multivariate rearrangements underlying dominance in terms of both curves. The area under each curve leads to a natural index of diagonal dependence. Interestingly, the average of both indices equals a multivariate generalization of Spearman's footrule and is closely related to the cograduation index proposed by Gini.
\end{abstract}

JEL-classification: D63, I31.

Keywords: Copula, Cumulative Deprivation, Cumulative Affluence, Diagonal Dependence, Spearman's Footrule, Gini's cograduation index.

*I thank Begoña Cabeza, Shaun Da Costa, Vanesa Jordá, Ana Perez Espartero, Mercedes Prieto Alaiz, Giovanna Scarchilli, and Mateo Séré for useful comments. Financial support of the Research Foundation - Flanders is gratefully acknowledged.

${ }^{\dagger}$ Centre for Social Policy (University of Antwerp), Department of Economics (KULeuven), Centre for Philosophy of Natural and Social Science (London School of Economics), and CORE (Université catholique de Louvain). 


\section{Introduction}

The contribution of Corrado Gini to the field of inequality measurement can hardly be overestimated. ${ }^{1}$ In his path-breaking work, Gini (1914b) introduced the concentration ratio as a new inequality measure and showed how it is linked to the Lorenz curve and to the mean absolute difference between all income pairs. This contribution spearheaded the statistical approach to inequality measurement. $^{2}$

The focus of Gini and his contemporaries was on the measurement of inequality in a single characteristic. Often, the characteristic of interest was a monetary variable such as income and wealth, but Gini (1921, p.124) stressed himself that "the same methods are, on the other hand, not only applicable to incomes and wealth, but to all other quantitative characteristics (economic, demographic, anatomical or physiological)". More recently, under the impetus of scholars like Amartya Sen $(1985 ; 1992)$, an interest has grown around measures of multidimensional inequality that summarize the inequality in multiple characteristics in a single measure. ${ }^{3}$

The main difference between a multidimensional approach to the measurement of inequality and a dimension-by-dimension analysis of each characteristic separately, is that the latter is not sensitive to the dependence between the characteristics (Decancq, 2014). Consider, for instance, a society where one individual is top-ranked according to all characteristics, another individual second-ranked, and so on. This society, reminiscent of a caste system, is arguably less equal than another society with exactly the same distributional profile in each characteristic separately, but where some individuals are performing relatively well on some characteristics and other individuals on others. Despite the difference in dependence between the dimensions, both societies will be judged to be at the same level of inequality by all dimension-by-dimension approaches.

\footnotetext{
${ }^{1}$ See Giorgi $(1990,1993)$ for (bibliographic) overviews of Gini's contribution to the field of inequality measurement.

${ }^{2}$ The statistical approach may be contrasted with the normative approach to the measurement of inequality, developed in the wake of Dalton (1920) and Atkinson (1970). This normative approach focuses on the social welfare cost of inequality, rather than on inequality itself.

${ }^{3}$ For a non-exhaustive overview of the literature on multidimensional inequality measurement, see Kolm (1977); Atkinson and Bourguignon (1982); Maasoumi (1986); Tsui (1995); Weymark (2006); and Bosmans et al. (2015). Andreoli and Zoli (forthcoming) provide an recent review and study how results about unidimensional stochastic dominance can be extended to the multidimensional setting.
} 
In this paper, I discuss the measurement of a specific aspect of dependence between dimensions of well-being, i.e., the cumulative deprivation and affluence. We say that there is more cumulative deprivation in a society when more persons occupy bottom positions in all dimensions of well-being. ${ }^{4}$ Several observers have argued that cumulative deprivation in itself is undesirable and unjust, see Decancq and Schokkaert (2016) for an overview. Similarly, there is more cumulative affluence in a society when more persons occupy top positions in all dimensions. While the notions of cumulative deprivation and affluence focus on different parts of the distribution, they are both concerned with the question of whether the positions in all characteristics are aligned. In other words, both notions focus on the dependence around the main diagonal of the multivariate distribution of positions. ${ }^{5}$

To quantify the dependence around the main diagonal, I follow an approach that echoes the Gini approach to inequality measurement. First, I introduce the diagonal dependence diagram, which is a graphical representation that consists of two curves: the downward and upward diagonal dependence curve. These curves are obtained by taking a diagonal section of the underlying copula and survival function, respectively. The downward diagonal dependence curve plays a role comparable to the Lorenz curve in inequality measurement and the upward diagonal dependence curve can be compared to the Leimkhuler curve (Sarabia, 2008). While the downward diagonal dependence curve summarizes the relevant information about cumulative deprivation, the upward diagonal dependence curve is useful to summarize cumulative affluence. Next, I study the elementary rearrangements that are equivalent to dominance in terms of these curves. These rearrangements play a role akin to that of Pigou-Dalton transfers in inequality measurement. Finally, I derive indices of diagonal dependence based on the area under the diagonal dependence curves. The average of the obtained indices of diagonal dependence equals Spearman's footrule, a measure of rank association that was proposed by Spearman in 1904. Interestingly, this measure is closely related to the cograduation index, the "indice di cograduazione semplice", which was originally proposed by Gini $(1914 \mathrm{a}, \S 98){ }^{6}$

\footnotetext{
${ }^{4}$ The notion of cumulative deprivation generalizes the intersection approach that is used in the analysis of multidimensional poverty, see Atkinson (2003); Alkire and Foster (2011); Aaberge et al. (2019).

${ }^{5}$ The main diagonal connects the position vector that consists of the bottom position in all dimensions with the position vector that consists of the top position in all dimensions.

${ }^{6}$ See Genest et al. (2010) for a historical overview of the use of Spearman's footrule and its link to Gini's cograduation index.
} 
The current work fits into a literature that uses copula-based techniques to measure dependence between dimensions of well-being in a scale-free manner. Early contributions by Quinn $(2007,2009)$ and (Atkinson, 2011) focus on the measurement of the dependence between health and income. Decancq (2014) looks at the dependence between income, health, and education in Russia, using non-parametric estimates of Spearman's $\rho$ and Kendall's $\tau$, and finds an increase in dependence between 1995 and 2005. Tkach and Gigliarano (2018) follow a parametric approach and derive Spearman's $\rho$ and Kendall's $\tau$ for several European countries. Pérez and Prieto (2015) refine the non-parametric measures used by Decancq (2014) and look at the dependence between income, material deprivation, and work intensity in Spain between 2009 and 2013. In a follow-up paper, García-Gómez et al. (2019) extend the analysis for 28 European countries between 2008 and 2014. Atkinson and Lakner (2017) consider the dependence between top labour and capital incomes in the United States between 1962 and 2006, and Aaberge et al. (2018) look at the case of Norway between 1995 and 2005. The paper by Pérez and Prieto-Alaiz (2016) is arguably closest to the current one in terms of dependence measures used. The authors look at the dependence between the dimensions of the Human Development Index using Spearman's footrule and Gini's cograduation index. They find that the dependence structure between these three dimensions is rather stable over time.

The contribution of the paper is threefold. First, it introduces the diagonal dependence diagram, which allows a parsimonious description of the dependence structure of a multivariate distribution around the diagonal with a onedimensional representation. The diagram provides a specific (geometric) interpretation of some existing abstract results from mathematical statistics and is closely related to the notion of tail dependence. The curves in the diagonal dependence diagram are useful to represent the cumulative deprivation and affluence in a society, but may be relevant for other applications as well (such as the measurement of multidimensional poverty). Second, the paper connects the curves of the diagonal dependence diagram to recent developments in multivariate stochastic dominance analysis, which provides additional insights into the underlying elementary rearrangements. Third, two natural measures of diagonal dependence are derived in this paper. These measures are closely related to Spearman's footrule and Gini's cograduation index.

The paper is structured as follows. Section 2 introduces the notation and presents the copula and survival functions as central building blocks. Section 
3 introduces the diagonal dependence diagram. Section 4 studies dominance in terms of the diagonal dependence curves. In Section 5 the diagonal dependence indices are derived and compared to other dependence measures. Section 6 concludes.

\section{The copula}

Let there be $m$ relevant dimensions of well-being such as income, health, social interactions, education, and so on. The random vector $X=\left(X_{1}, \ldots, X_{m}\right)$ describes the distribution of the outcomes in these dimensions across the individuals in a society. The cumulative distribution function of the random vector $X$ is denoted $F_{X}$. For all $x=\left(x_{1}, \ldots, x_{m}\right)$, we have:

$$
F_{X}\left(x_{1}, \ldots, x_{m}\right)=\operatorname{Pr}\left[X_{1} \leq x_{1} \text { and } \ldots \text { and } X_{m} \leq x_{m}\right]
$$

For each realization of the random vector, the cumulative distribution function returns the proportion of individuals in the society who have less or equal in each dimension of well-being. Similarly, $\bar{F}_{X}$, the decumulative distribution function or survival function, returns for each realization of the random vector the proportion of individuals in the society who have strictly more in every dimension. For all $x=\left(x_{1}, \ldots, x_{m}\right)$, we have:

$$
\bar{F}_{X}\left(x_{1}, \ldots, x_{m}\right)=\operatorname{Pr}\left[X_{1}>x_{1} \text { and } \ldots \text { and } X_{m}>x_{m}\right] .
$$

In contrast to the univariate case, the equation $F_{X}=1-\bar{F}_{X}$ is generally not true in the multivariate case.

The marginal distribution function of dimension $j=1, \ldots, m$ is denoted $F_{j}$. In what follows, the position of the individual having $x_{j}$ in dimension $j$, will be referred to as $p_{j}=F_{j}\left(x_{j}\right)$. A position belongs to the set $\mathbb{I} \subseteq[0,1]$ and may be interpreted as the rank in the distribution of the individual having $x_{j}$. The position vector $p=\left(p_{1}, \ldots, p_{m}\right)$ of an individual lists their positions in all dimensions of well-being. An individual with a position vector equal to $(1, \ldots, 1)$ is top-ranked in all dimensions, whereas an individual with position vector $(0, \ldots, 0)$ is bottom-ranked. One position vector is said to be outranked by another if it has a lower or equal position in all dimensions. Conversely, one position vector outranks another if it has a higher position in all dimensions. 
The random variable $P_{j}=F_{j}\left(X_{j}\right)$, describes the distribution of the positions in the $j$ th dimension of well-being and follows a standard uniform distribution. The random vector $P(X)=\left(F_{1}\left(X_{1}\right), \ldots, F_{m}\left(X_{m}\right)\right)$ captures the distribution and alignment of the positions in the society. The cumulative distribution function of the positions $F_{P(X)}$ is a copula function, and will be denoted $C_{X} \cdot{ }^{7}$ A copula function is an $m$-dimensional cumulative distribution function whose support is $\mathbb{I}^{m}$ and whose one-dimensional marginal distribution functions follow a standard uniform distribution. The copula function of random vector $X$ returns for every position vector the proportion of individuals in the society who are outranked by the position vector. Hence, for all $p=\left(p_{1}, \ldots, p_{m}\right)$ in $\mathbb{I}^{m}$, we have:

$$
C_{X}\left(p_{1}, \ldots, p_{m}\right)=\operatorname{Pr}\left[F_{1}\left(X_{1}\right) \leq p_{1} \text { and } \ldots \text { and } F_{m}\left(X_{m}\right) \leq p_{m}\right]
$$

The more the position vectors are aligned, the larger the proportion of individuals in the society being outranked, and the larger the copula function for any given position vector.

The survival function $\bar{C}_{X}$ can be defined in an analogous way. It returns, for every position vector, the proportion of individuals in the society who are outranking the position vector. For all $p=\left(p_{1}, \ldots, p_{m}\right)$ in $\mathbb{I}^{m}$, we have:

$$
\bar{C}_{X}\left(p_{1}, \ldots, p_{m}\right)=\operatorname{Pr}\left[F_{1}\left(X_{1}\right)>p_{1} \text { and } \ldots \text { and } F_{m}\left(X_{m}\right)>p_{m}\right]
$$

In the multivariate case, the copula function $C_{X}$ and the survival function $\bar{C}_{X}$ offer a complementary view on the alignment of the position vectors. While the copula function looks "downwards" at the proportion of individuals who are outranked, the survival function looks "upwards" at the proportion of individuals who are outranking. The copula function will turn out to be a useful tool to describe the phenomenon of cumulative deprivation, while the survival function is useful to describe cumulative affluence.

The popularity of the copula function can be largely attributed to the result by Sklar (1959) that shows that every multivariate distribution function $F_{X}$ can be written as a function of its $m$ marginal distribution functions $F_{j}$ and its copula function $C_{X}$ :

$$
F_{X}\left(x_{1}, \ldots, x_{m}\right)=C_{X}\left(F_{1}\left(x_{1}\right), \ldots, F_{m}\left(x_{m}\right)\right) .
$$

\footnotetext{
${ }^{7}$ See Nelsen (2006) and Trivedi and Zimmer (2007) for a thorough discussion of the copula function and its properties.
} 
Moreover, if $F_{1}, \ldots, F_{m}$ are all continuous, $C_{X}$ is unique; otherwise $C_{X}$ is uniquely determined on $\mathbb{I}^{m}=$ Range $F_{1} \times \cdots \times$ Range $F_{m}$. Sklar's result permits a decomposition of the $m$-dimensional distribution function into its marginal distribution functions and its copula function. Standard (one-dimensional) inequality measures such as the Gini coefficient can be used to quantify the inequality in the marginal distributions, while copula-based measures of dependence allows one to summarize the dependence structure.

The independence copula $C_{\perp}\left(p_{1}, \ldots, p_{m}\right)=p_{1} \times \cdots \times p_{m}$ is an intuitive example of a copula function. By substituting $p_{j}=F_{j}\left(x_{j}\right)$, the independence joint distribution function can be retrieved as the product of its marginal distribution functions. When the distribution of the position vectors in a society is given by the independence copula, the dimensions of well-being are independent. The political philosopher Michael Walzer (1983) describes such a society as a complex equal society (see also Miller (1995)).

Every copula function is bounded by its Fréchet-Hoeffding bounds, which are defined by $C_{-}\left(p_{1}, \ldots, p_{m}\right)=\max \left(p_{1}+\cdots+p_{m}-m+1,0\right)$ and $C_{+}\left(p_{1}, \ldots, p_{m}\right)=$ $\min \left(p_{1}, \ldots, p_{m}\right)$ respectively (Nelsen, 2006, p.11). ${ }^{8}$ Hence, for all $p=\left(p_{1}, \ldots, p_{m}\right)$ in $\mathbb{I}^{m}$, we have:

$$
C_{-}\left(p_{1}, \ldots, p_{m}\right) \leq C_{X}\left(p_{1}, \ldots, p_{m}\right) \leq C_{+}\left(p_{1}, \ldots, p_{m}\right)
$$

The Fréchet-Hoeffding bounds $C_{-}$and $C_{+}$will be referred to as the countermonotonic and comonotonic case. In the countermonotonic case $C_{-}$, high ranks in some dimensions come with low ranks in others, and none of the position vectors outrank or is outranked by any other position vector. In the comonotonic case $C_{+}$, on the other hand, there is one individual who is top-ranked in all dimensions of well-being, another individual second-ranked in all dimensions, and so on.

Figure 1 shows contour diagrams of $C_{-}, C_{\perp}$, and $C_{+}$in the bivariate case. Between two contour curves in the diagram, ten percent of the individuals in the society can be found. While all individuals can be found on the antidiagonal that connects the position vectors $(0,1)$ and $(1,0)$ in the first case, they are evenly scattered around in the second case, and are all situated on the main diagonal between the position vectors $(0,0)$ and $(1,1)$ in the latter case. The

\footnotetext{
${ }^{8}$ For $m \geq 3$, the function $C_{-}$is a lower bound for the copula function, but it is not a distribution function and, hence, cannot be a copula function (Nelsen, 2006, p. 47).
} 
contour curves are linear for $C_{-}$, are curved for $C_{\perp}$, and kinked for $C_{+}$. In all three cases, the marginal distributions of the positions follow a standard uniform distribution.
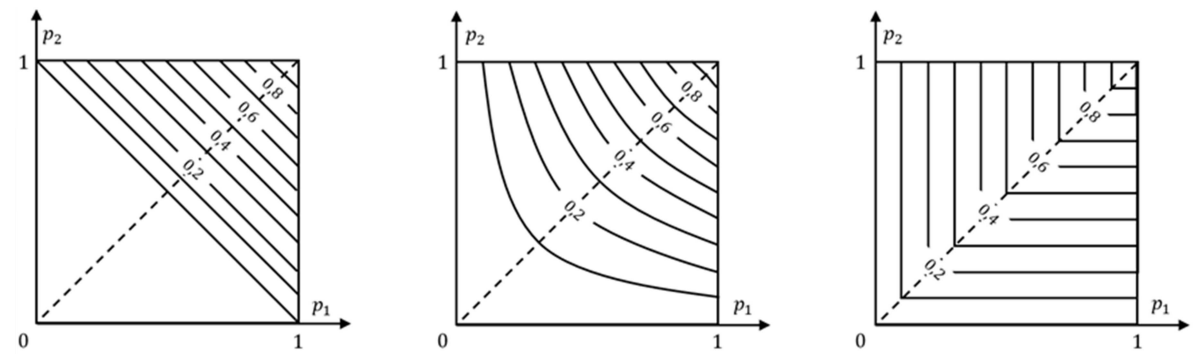

Figure 1: Contour diagrams of $C_{-}, C_{\perp}$, and $C_{+}$

\section{The diagonal dependence diagram}

In this section, the diagonal dependence diagram is introduced. It consists of two curves: the downward and upward diagonal dependence curve. These curves are derived from the copula and survival function, respectively.

The contour diagrams of Figure 1 are useful to introduce the downward diagonal dependence curve. In every panel the main diagonal is indicated with a dashed line. The downward diagonal dependence curve is obtained by making a section of the copula function along that diagonal. ${ }^{9}$

Definition 1 (Downward Diagonal Dependence Curve). For all $\mathrm{m}$ dimensional random vectors $X$ with copula function $C_{X}$, we say that $D_{X}$ is the downward diagonal dependence curve of $X$, if for all $p$ in $\mathbb{I}$ :

$$
D_{X}(p)=C_{X}(p, \ldots, p) .
$$

For each position $p$, the downward diagonal dependence curve gives the proportion of the society who have a lower or equal position than $p$ in all dimensions

\footnotetext{
${ }^{9}$ In mathematical statistics, the downward diagonal dependence curve is known as the diagonal section of a copula function, see Nelsen (2006, p.12). Fernández-Sánchez and ÚbedaFlores (2018) give a recent review of the statistical literature that deals with the existence and properties of the copula for a given diagonal section.
} 
of well-being. The proportion of the society which is below or equal to the median in all dimensions is given by $D_{X}(0.5)$, for instance. The curve represents the local information about dependence around the main diagonal and takes a "downward" perspective, by focusing on the position vectors with a lower or equal position than the position vectors on the diagonal.

The left-hand panel of Figure 2 gives an example of a downward diagonal dependence curve for a multivariate random vector $X$. The dashed line gives the downward diagonal dependence curve of the comonotonic case and is denoted $D_{+}$. The downward diagonal dependence curve of an independent $m$ dimensional distribution equals $(1 / p)^{m}$ and is denoted $D_{\perp}$. Every downward diagonal dependence curve starts at 0 (when $p=0$ ) and ends at 1 (when $p=1$ ). Moreover, we have that $D_{X}(p) \leq p$ for all random vectors $X$.
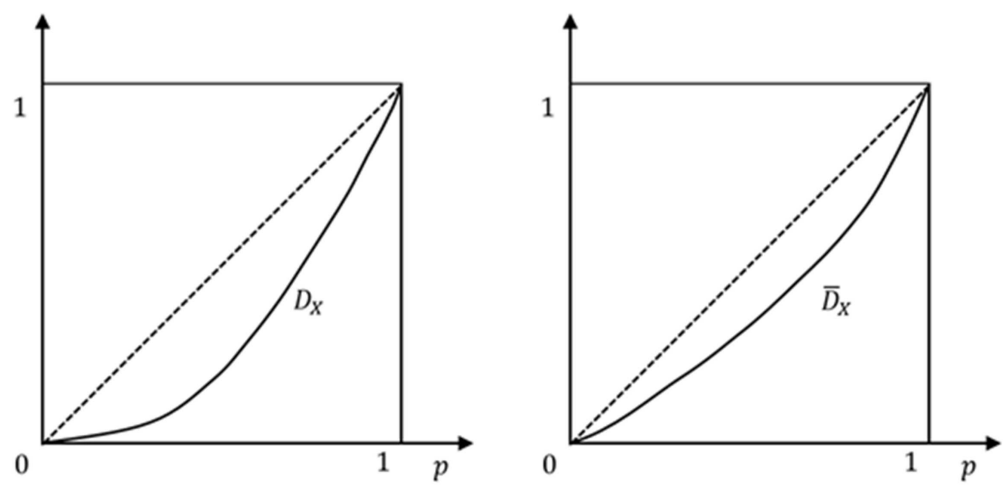

Figure 2: The downward diagonal dependence curve $D_{X}$ (left) and upward diagonal dependence curve $\bar{D}_{X}$ (right) of random vector $X$

Analogous to the derivation of the downward diagonal dependence curve $D_{X}$ from the copula function $C_{X}$, the upward diagonal dependence curve $\bar{D}_{X}$ can be derived from the survival function $\bar{C}_{X}$.

Definition 2 (Upward Diagonal Dependence Curve). For all $m$-dimensional random vectors $X$ with survival function $\bar{C}_{X}$, we say that $\bar{D}_{X}$ is the upward diagonal dependence curve of $X$, if for all $p$ in $\mathbb{I}$ :

$$
\bar{D}_{X}(p)=\bar{C}_{X}(1-p, \ldots, 1-p) .
$$

For each position $p$, the upward diagonal dependence curve gives the proportion of the society who have a higher position than $1-p$ in all dimensions of 
well-being. The proportion of the society which belongs to the top decile in all dimensions is given by $\bar{D}_{X}(0.1)$, for instance. While the downward diagonal dependence curve presents the "downward" perspective of the copula function, the upward diagonal dependence curve presents the "upward" perspective of the survival function. In general, both diagonal dependence curves do not coincide. ${ }^{10}$ The right-hand panel of Figure 2 gives an example of an upward diagonal dependence curve. The dashed line shows the upward diagonal dependence curve of the comonotonic case $C_{+}$.

Conveniently, both diagonal dependence curves can be plotted in one diagram, the diagonal dependence diagram (see Figure 3), by rotating the upward diagonal dependence curve $\bar{D}_{X}$ (the right-hand panel of Figure 2) $180^{\circ}$ and superimposing it on the downward diagonal dependence curve $D_{X}$ (the left-hand panel of Figure 2). The downward diagonal dependence curve uses the origin in the south-west and the upward diagonal dependence curve uses the origin in the north-east.

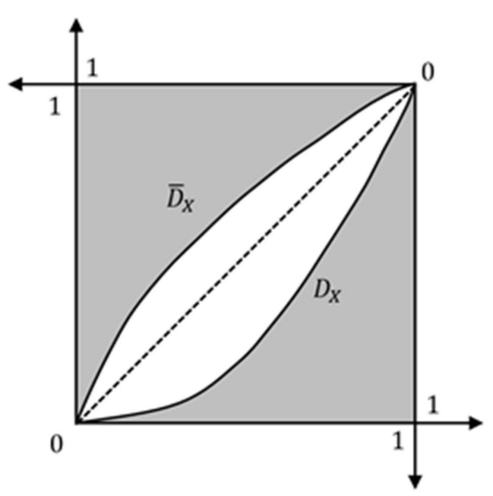

Figure 3: The diagonal dependence diagram

The diagonal dependence diagram contains information about the local dependence around the main diagonal, both from an upward and downward perspective. For some applications, such as the measurement of cumulative deprivation and affluence, the focus on the local dependence information around the diagonal is warranted or even desired. For other applications, this may not be the case. Compared to its underlying copula and survival functions, the diagonal depend-

\footnotetext{
${ }^{10}$ Both curves coincide for random vectors which exhibit radial symmetry about $(1 / 2, \ldots, 1 / 2)$, so that $C_{X}(p, \ldots, p)=\bar{C}_{X}(1-p, \ldots, 1-p)$ for all $p=\left(p_{1}, \ldots, p_{m}\right)$ in (Nelsen, 2006, p.36).
} 
ence diagram has the advantage of being one-dimensional and, hence, easier to visualize and represent. This practical advantage comes at a price, however, as information "off" the main diagonal is not contained in the diagram. We will return to this observation in the next section.

Finally, the curves in the diagonal dependence diagram are linked to the tail dependence parameters, which focus on local dependence information around the diagonal when $p$ approaches 0 (Joe, 1997; Nelsen, 2006; Schmid and Schmidt, 2007a). In the bivariate case, the lower tail dependence parameter $\lambda_{L}$ is the limit of the conditional probability that an individual has a position in dimension $j_{1}$ which is lower than or equal to $p$, given that she has a position in dimension $j_{2}$ which is lower than or equal to $p$, as $p$ approaches 0 . The upper tail dependence parameter $\lambda_{U}$, on the other hand, is the limit of the conditional probability that an individual has a position in dimension $j_{1}$ which is higher than $p$, given that she has a position in dimension $j_{2}$ which is higher than $p$, as $p$ approaches 1 . Nelsen (2006, Theorem 5.4.2) shows in the bivariate case how both tail dependence parameters are related to the diagonal dependence curves, respectively:

$$
\lambda_{L}=\lim _{p \rightarrow 0^{+}} \frac{D_{X}(p)}{p} \text { and } \lambda_{U}=\lim _{p \rightarrow 1^{-}} \frac{\bar{D}_{X}(1-p)}{1-p}=\lim _{q \rightarrow 0^{+}} \frac{\bar{D}_{X}(q)}{q}
$$

In other words, the tail dependence parameters capture how close the diagonal dependence curves of a random vector $X$ are to the diagonal when $p$ approaches 0 . The closer the limit is to the main diagonal, the more tail dependence the random vector exhibits. Tail dependence parameters are useful to study cumulative deprivation or affluence at the very bottom or top of the distribution, when the focus is either on the poorest or on the most affluent in the society.

\section{The diagonal dependence ordering}

In this section, I define first the downward and upward diagonal dependence ordering based on dominance of the curves in the diagonal dependence diagram. Second, the elementary multivariate rearrangements that underlie these orderings are studied.

If the downward diagonal dependence curve of random vector $Y$ is everywhere above the downward diagonal dependence curve of $X$, i.e., when the curve of $Y$ is closer to the $45^{\circ}$ line, we say that $Y$ dominates $X$ according to the downward 
diagonal dependence ordering.

Definition 3 (Downward Diagonal Dependence Ordering). For all $\mathrm{m}$ dimensional random vectors $X$ and $Y$ with downward diagonal dependence curves $D_{X}$ and $D_{Y}$, we say that $Y$ dominates $X$ according to the downward diagonal dependence ordering, if $D_{Y}(p) \geq D_{X}(p)$ for all $p$ in $\mathbb{I}$.

In other words, the random vector $Y$ dominates the random vector $X$ according to the downward diagonal dependence ordering if the copula function of $Y$ is above the copula function of $X$ for all position vectors on the main diagonal. The latter restriction on the considered position vectors makes the downward diagonal dependence an ordering of local dependence around the main diagonal and considerably weaker than the lower orthant dependence ordering, which can be seen as an ordering of global dependence and requires that the copula function of $Y$ is above or equal to the copula function of $X$ for all position vectors.

In fact, the test of non-intersecting downward diagonal dependence curves, which is required for the downward diagonal dependence ordering, looks formally similar to the test for the univariate first order stochastic dominance ordering, which requires that the univariate cumulative distribution functions do not intersect. Thanks to this similarity, statistical tests developed for first order stochastic dominance can be used to test for the downward diagonal dependence ordering as well, see Cowell and Flachaire (2015) for a recent overview.

Analogously, we say that $Y$ dominates $X$ according to the upward diagonal dependence ordering, if the upward diagonal dependence curve of random vector $Y$ is everywhere above the upward diagonal dependence curve of $X$.

Definition 4 (Upward Diagonal Dependence Ordering). For all $\mathrm{m}^{-}$ dimensional random vectors $X$ and $Y$ with upward diagonal dependence curves $\bar{D}_{X}$ and $\bar{D}_{Y}$, we say that $Y$ dominates $X$ according to the upward diagonal dependence ordering, if $\bar{D}_{Y}(p) \geq \bar{D}_{X}(p)$ for all $p$ in $\mathbb{I}$.

The upward diagonal dependence ordering requires the survival function of $Y$ to be above the survival function of $X$ for all position vectors on the main diagonal. Thus, it is an ordering of local dependence around the diagonal. It is a weakening of the upper orthant dependence ordering which requires the survival function of $Y$ to be everywhere above or equal to the survival function of $X^{11}$

${ }^{11}$ Echoing the similarity between the downward diagonal dependence ordering and univari- 
In the bivariate case, both dependence orderings are equivalent. ${ }^{12}$ Yet, this equivalence does not hold when $m>2$. While the downward diagonal dependence ordering takes a downward perspective, the upward diagonal dependence ordering takes an upward perspective to diagonal dependence. Both perspectives are combined in the following ordering.

Definition 5 (Diagonal Dependence Ordering). For all $m$-dimensional random vectors $X$ and $Y$ with downward diagonal dependence curves $D_{X}$ and $D_{Y}$, and upward diagonal dependence curves $\bar{D}_{X}$ and $\bar{D}_{Y}$, we say that $Y$ dominates $X$ according to the diagonal dependence ordering, if $D_{Y}(p) \geq D_{X}(p)$ and $\bar{D}_{Y}(p) \geq \bar{D}_{X}(p)$ for all $p$ in $\mathbb{I}$.

The diagonal dependence ordering focuses on local dependence information around the main diagonal, irrespective of the choice for an upward or downward perspective. It is a weakening of the concordance ordering proposed by Joe (1990), which combines the lower and upper orthant orders and requires dominance in terms of the copula and survival functions.

To evaluate whether the diagonal dependence orderings are attractive orderings for our purpose of measuring cumulative deprivation and affluence, it is useful to consider the underlying elementary rearrangements. Elementary rearrangements that lead to more global dependence have been studied in the bivariate case by Tchen (1980) and Epstein and Tanny (1980), following a suggestion by Hamada (1974). Atkinson and Bourguignon (1982) provide an application for the measurement of multidimensional inequality. The considered rearrangements shift probability mass $\varepsilon$ on the four vertices of a two-dimensional rectangle (see Figure 4). A positive probability mass $\varepsilon$ is shifted from the two white vertices with an odd number of 'lows', that is, the vertices (low, high) and (high, low) to the two black vertices with an even number of 'lows', that is, (low, low) and (high, high). ${ }^{13}$ After the rearrangement (the black situation on Figure 4), individuals at (low, low) are outranked by individuals at (high, high) and similarly individuals at (high, high) outrank individuals at (low, low), whereas in the initial (white) situation, the considered individuals do not outrank each other. Hence, the copula and survival function have shifted upwards. Tchen (1980) and Epstein and Tanny (1980) show that the converse is also true in the

ate first order stochastic dominance, the upward diagonal dependence ordering is formally similar to the univariate first order decumulative stochastic dominance discussed by Bazen and Moyes (2012).

${ }^{12}$ When $m=2$, we have that $\bar{C}_{X}(p, p)=1-2 p+C_{X}(p, p)$.

${ }^{13}$ In the following, I adopt the convention that 0 is even. 
bivariate case: whenever the copula function (or survival function) of random vector $Y$ is everywhere above the one of random vector $X$, random vector $Y$ can be obtained from $X$ by a sequence of rearrangements on the corners of a two-dimensional rectangle.

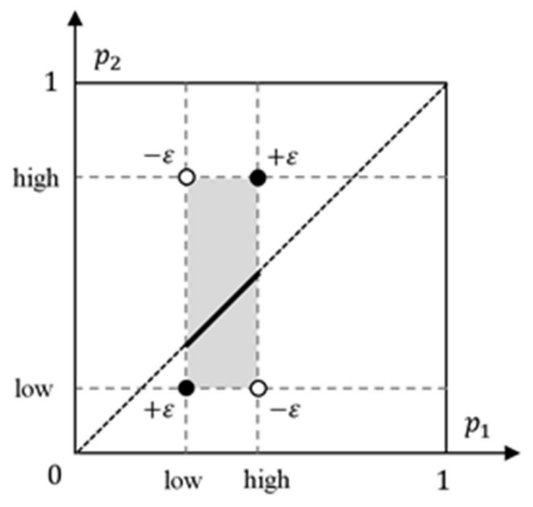

Figure 4: A positive 2-rearrangement

Decancq (2012) presents a multivariate generalization of the results by Tchen (1980) and Epstein and Tanny (1980). He considers elementary rearrangements that shift probability mass $\varepsilon$ on the $2^{k}$ vertices of a $k$-dimensional hyperbox, with $2 \leq k \leq m$. The probability mass is shifted from the $2^{k-1}$ vertices with an odd number of 'lows' to the $2^{k-1}$ vertices with an even number of 'lows'. He defines positive $k$-rearrangements, for which a positive probability mass $\varepsilon>0$ is shifted, and alternating $k$-rearrangements, for which $(-1)^{k} \varepsilon>0$ holds. ${ }^{14}$ The two-dimensional example given in Figure 4 can be referred to as a positive 2rearrangement (or an alternating 2-rearrangement). When $k$ is even, a positive $k$-rearrangement is also an alternating $k$-rearrangement, and vice versa. This equality does not hold when $k$ is odd.

Table 1 gives an example of a positive 3-rearrangement (when $\varepsilon>0$ ). While this rearrangement shifts positive probability mass $\varepsilon$ to the position vector ( high, high, high), probability mass $\varepsilon$ is removed from the position vector (low, low, low). After the rearrangement, more individuals outrank other individuals, while less individuals are outranked. As the probability of outranking has increased after the rearrangement, the survival function shifts upwards, while the copula function shifts downwards (since the probability of being outranked has decreased).

\footnotetext{
${ }^{14}$ Müller (2013) refers to these rearrangements as $\Delta$-monotone transfers and $\Delta$-antitone transfers, respectively.
} 
In general, Decancq (2012) shows that alternating $k$-rearrangements underlie the lower orthant dependence ordering (i.e., dominance in terms of the copula function), while positive $k$-rearrangements underlie the upper orthant dependence ordering (i.e., dominance in terms of the survival function), see Müller (2013) and Decancq (2014) for further discussions.

\begin{tabular}{cc}
\hline$-\varepsilon$ & $+\varepsilon$ \\
\hline (low, high, high $)$ & $($ high, high, high $)$ \\
(high, low, high $)$ & $($ high, low, low $)$ \\
(high, high, low $)$ & $($ low, high, low $)$ \\
(low, low, low $)$ & $($ low, low, high $)$ \\
\hline
\end{tabular}

Table 1: A positive 3-rearrangement (when $\varepsilon>0$ )

The described elementary rearrangements on the corners of $k$-dimensional hyperbox affect (global) dependence as measured by the orthant orderings. They may or may not affect the diagonal dependence curves, which only capture local dependence information around the main diagonal. Only if the main diagonal intersects the hyperbox, as on the highlighted segment of the main diagonal in Figure 4, will the diagonal dependence curves be affected by the rearrangement. Rearrangements that happen "off the diagonal", on the other hand, will not affect the diagonal dependence curves. Conversely, when there is dominance between the diagonal dependence curves, there exists a sequence of $k$-rearrangements on the vertices of a $k$-dimensional hyperbox, which are intersected by the main diagonal.

\section{The diagonal dependence index}

Based on the area below both curves in the diagonal dependence diagram (i.e., the shaded area of Figure 3), indices of diagonal dependence can be derived in a natural way.

First, however, I present an intuitive interpretation of the area below the diagonal dependence curves. We have that: ${ }^{15}$

$$
\int_{\mathbb{I}} D_{X}(p) d p=\int_{\mathbb{I}^{m}} C_{X}\left(p_{1}, \ldots, p_{m}\right) d C_{+}\left(p_{1}, \ldots, p_{m}\right)
$$

${ }^{15}$ See Úbeda-Flores (2005, Lemma 4.1) for a formal proof. 
and

$$
\int_{\mathbb{I}} \bar{D}_{X}(p) d p=\int_{\mathbb{I}^{m}} \bar{C}_{X}\left(p_{1}, \ldots, p_{m}\right) d C_{+}\left(p_{1}, \ldots, p_{m}\right) .
$$

In other words, the area under the downward diagonal dependence curve equals the probability that a randomly selected individual in the society described by random vector $X$ is outranked by a randomly selected individual from a perfectly comonotonic society, described by $X_{+}$. The more cumulative deprivation there is in $X$ (according to the downward diagonal dependence ordering), the larger this probability becomes. The area under the upward diagonal dependence curve, on the other hand, equals the probability that a randomly selected individual in $X$ outranks a randomly selected individual from a perfectly comonotonic society. The sum of both areas equals the probability of concordance between the random vector $X$ and $X_{+}$(i.e., the probability that a realization of $X$ is outranked by or outranks a realization of $\left.X_{+}\right)$.

We start by defining the downward diagonal dependence index, denoted $\delta_{m}^{-}$, based on the area below the downward diagonal dependence curve.

Definition 6 (Downward Diagonal Dependence Index). For all $\mathrm{m}$ dimensional random vectors $X$ with downward diagonal dependence curve $D_{X}$, we say that $\delta_{m}^{-}(X)$ is the downward diagonal dependence index of $X$, if

$$
\delta_{m}^{-}(X)=\frac{2(m+1) \int_{\mathbb{I}} D_{X}(p) d p-2}{m-1} .
$$

The downward diagonal dependence index is based on the area below the downward diagonal dependence curve, normalized such that $\delta_{m}^{-}\left(X_{\perp}\right)=0$ and $\delta_{m}^{-}\left(X_{+}\right)=$ $1{ }^{16}$ As we have seen, the index can be interpreted as the normalized probability that a randomly selected individual in $X$ is outranked by a randomly selected individual from $X_{+}$. If $X$ and $Y$ are two random vectors with $D_{Y}(p) \geq D_{X}(p)$ for all $p$ in $\mathbb{I}$, then we have that $\delta_{m}^{-}(Y) \geq \delta_{m}^{-}(X)$. Hence, the downward diagonal dependence index is consistent with the downward diagonal dependence ordering (as well as with the lower orthant dependence ordering).

Similarly, the upward diagonal dependence index, $\delta_{m}^{+}$, can be defined based on the normalized area below the upward diagonal dependence curve.

\footnotetext{
${ }^{16}$ This can be checked by observing that $\int_{\mathbb{I}} D_{\perp}(p) d p=1 /(m+1)$ and $\int_{\mathbb{I}} D_{+}(p) d p=1 / 2$.
} 
Definition 7 (Upward Diagonal Dependence Index). For all $m$-dimensional random vectors $X$ with upward diagonal dependence curve $\bar{D}_{X}$, we say that $\delta_{m}^{+}(X)$ is the upward diagonal dependence index of $X$, if

$$
\delta_{m}^{+}(X)=\frac{2(m+1) \int_{\mathbb{I}} \bar{D}_{X}(p) d p-2}{m-1} .
$$

The upward diagonal dependence index $\delta_{m}^{+}$equals the normalized probability that a randomly selected individual in $X$ outranks a randomly selected individual from $X_{+}$. Similar to the downward diagonal dependence index, we have that $\delta_{m}^{+}\left(X_{\perp}\right)=0$ and $\delta_{m}^{+}\left(X_{+}\right)=1$. The upward diagonal dependence index is consistent with the upward diagonal dependence ordering (and the upper orthant dependence ordering).

While the downward diagonal dependence index has a "downward" perspective that measures how often individuals are outranked by others, the upward diagonal dependence index has an "upward" perspective that measures how often individuals outrank others. When the context at hand does not warrant a specific focus on either perspective, both deprivation indices can be combined by averaging them.

Definition 8 (Diagonal Dependence Index). For all $m$-dimensional random vectors $X$ with downward diagonal dependence curve $D_{X}$ and upward diagonal dependence curve $\bar{D}_{X}$, we say that $\delta_{m}(X)$ is the diagonal dependence index of $X$, if

$$
\delta_{m}(X)=\frac{\delta_{m}^{-}(X)+\delta_{m}^{+}(X)}{2}=\frac{(m+1) \int_{\mathbb{I}}\left[D_{X}(p)+\bar{D}_{X}(p)\right] d p-2}{m-1} .
$$

For the diagonal dependence index $\delta_{m}$, we also have that $\delta_{m}\left(X_{\perp}\right)=0$ and $\delta_{m}\left(X_{+}\right)=1$. The diagonal dependence index is consistent with the diagonal dependence ordering as well as the concordance dependence ordering.

While, to the best of my knowledge, the downward and upward diagonal dependence indices $\delta_{m}^{-}$and $\delta_{m}^{+}$are new to the literature, the diagonal dependence index $\delta_{m}$ is not. It equals the multidimensional generalization of Spearman's footrule which has been proposed by Úbeda-Flores (2005, Theorem 4.1) and which is commonly denoted $\varphi_{m} \cdot{ }^{17}$

\footnotetext{
${ }^{17}$ See Úbeda-Flores (2005), Genest et al. (2010), and Pérez and Prieto-Alaiz (2016) for more extensive discussions of Spearman's footrule.
} 
The multidimensional generalization of Spearman's footrule proposed by ÚbedaFlores (2005) belongs to the class of dependence measures that are based on the probability of concordance between $X$, the random vector at hand, and a reference random vector. This class has been introduced by Nelsen (1991, 2002), and Decancq (2014) interprets it in the context of measuring dependence between dimensions of well-being. Specifically, Spearman's footrule is derived from the probability of concordance between $X$ and a comonotonic reference vector $X_{+}$. In a similar way, other popular dependence measures can be obtained from the probability of concordance between $X$ and other reference random vectors, such as Spearman's $\rho_{m}$ (when the reference random vectors equals $X_{\perp}$ ) or Kendall's $\tau_{m}$ (when the reference random vector equals $X$ itself), see Nelsen $(2002,2006)$ and Decancq (2014).

In the bivariate case, the three diagonal dependence indices are equal (i.e., $\delta_{2}=\delta_{2}^{-}=\delta_{2}^{+}$). When $m>2$, however, this is not the case. Some societies may exhibit more downward diagonal dependence (or cumulative deprivation) and little upward diagonal dependence (or cumulative affluence), or vice versa. Consider, for instance, the two four-person societies described by the position vectors which are listed in the two columns of Table 1 . While there is more cumulative deprivation in the society described by the left-hand column, there is more cumulative affluence in the society described by the right-hand column. A decomposition of Spearman's footrule in the downward and upward diagonal dependence indices with their complementary upward and downward perspectives can therefore lead to additional new insights. ${ }^{18}$

In view of empirical applications, it is useful to note that Úbeda-Flores (2005, p. 786) has suggested an easy-to-implement multivariate sample version of Spearman's footrule $\varphi_{m}$ (and, hence, of the diagonal dependence index $\delta_{m}$ ). Let $r_{i j}$ be the rank of outcome $x_{i j}$ of individual $i=1, \ldots, n$ in dimension $j=1, \ldots, m$. Spearman's footrule can be estimated by

$$
\widehat{\varphi}_{m}(X)=1-\frac{m+1}{m-1} \frac{\sum_{i=1}^{n}\left[\max _{j}\left(r_{i j}\right)-\min _{j}\left(r_{i j}\right)\right]}{n^{2}-1} .
$$

Genest et al. (2010) show that $\widehat{\varphi}_{m}$ is an asymptotically unbiased estimator of $\varphi_{m}$, with an asymptotic normal distribution. The bivariate version of expression (13) was originally proposed in the work of Spearman (1904), who discussed the

\footnotetext{
${ }^{18}$ Pérez and Prieto (2015) advocate the use of an analogous decomposition of Spearman's $\rho$ in a "downward" measure $\rho^{-}$and an "upward" measure $\rho^{+}$, which have been discussed by Joe (1990), Nelsen (1996), and Schmid and Schmidt (2007b).
} 
following measure of rank association:

$$
\widehat{\varphi}_{2}\left(X_{1}, X_{2}\right)=1-\frac{3 \sum_{i=1}^{n}\left|r_{i 1}-r_{i 2}\right|}{n^{2}-1} .
$$

Spearman himself noted that one of the disadvantages of the proposed index is that it is not symmetric under the reversal of one dimension. ${ }^{19}$ To remedy this asymmetry, Salama and Quade (2001) have proposed a symmetric version of the index by considering the average of the footrule index and the footrule index that is obtained by reversing the ranking of one variable. Interestingly, Nelsen and Úbeda-Flores (2004) have remarked that the proposed symmetric version of the footrule index coincides with Gini's cograduation index $\gamma_{m}$ (Gini, 1914a, $\S 98)$. The bivariate sample version of Gini's cograduation index, $\widehat{\gamma}_{2}$, equals

$$
\widehat{\gamma}_{2}\left(X_{1}, X_{2}\right)=\frac{\sum_{i=1}^{n}\left[\left|n+1-r_{i 1}-r_{i 2}\right|-\left|r_{i 1}-r_{i 2}\right|\right]}{\left\lfloor n^{2} / 2\right\rfloor} .
$$

where $\lfloor d\rfloor$ denotes the integer part of $d$ with $d>0$. Behboodian et al. (2007) have proposed a multivariate extension of Gini's cograduation index $\gamma$ that belongs to the class of dependence measures that are derived from the probability of concordance, by choosing the reference random vector equal to the average of the countermonotonic $X_{-}$and comonotonic case $X_{+}$.

\section{Conclusion}

In the wake of Gini's path-breaking work on inequality measurement more than a century ago, a plethora of inequality measures have been developed, which leaves researchers with a difficult task when choosing the most appropriate measure. A rigorous axiomatic study of the properties that uniquely characterize these inequality measures helps to make this choice. A similar problem arises for researchers who have to select the most appropriate dependence measure. The answer depends largely on the application and research question at hand. When a global perspective on dependence is required, dependence measures such as Kendall's $\tau_{m}$ and Spearman's $\rho_{m}$ seem most appropriate. When a local perspective focusing on the dependence around the main diagonal is required, however, Spearman's footrule and the diagonal dependence indices that are introduced

\footnotetext{
${ }^{19}$ More recently, Dolati and Úbeda-Flores (2006) and Taylor (2007) discuss this symmetry as a desirable property of a multivariate measure of concordance.
} 
in this paper are useful. When a local but symmetric perspective with respect to both diagonals is required, Gini's cograduation index $\gamma_{m}$ appears to be a good choice. In general, however, there seems to be ample room for additional axiomatic work on the question of which properties uniquely characterize these dependence measures. Studying how the dependence measures react to specific elementary rearrangements could be a point of departure for this endeavour.

\section{References}

Aaberge, R., A. B. Atkinson, and S. Königs (2018): "From classes to copulas: wages, capital, and top incomes," Journal of Economic Inequality, $16,295-320$.

Aaberge, R., E. Peluso, and H. Sigstad (2019): "The dual approach for measuring multidimensional deprivation: Theory and empirical evidence," Journal of Public Economics, 177, 104036.

Alkire, S. And J. Foster (2011): "Counting and multidimensional poverty measurement," Journal of Public Economics, 95, 476-487.

Andreoli, F. And C. Zoli (forthcoming): "From unidimensional to multidimensional inequality: a review," Metron.

Atkinson, A. B. (1970): "On the measurement of inequality," Journal of Economic Theory, 2, 244-263.

- (2003): "Multidimensional deprivation: contrasting social welfare and counting approaches," Journal of Economic Inequality, 1, 51-65.

(2011): "On lateral thinking," Journal of Economic Inequality, 9, 319328.

Atkinson, A. B. And F. Bourguignon (1982): "The comparison of multidimensioned distributions of economic status," The Review of Economic Studies, 49, 183-201.

Atkinson, A. B. And C. Lakner (2017): "Capital and Labor: The Factor Income Composition of Top Incomes in the United States, 1962-2006," World Bank Policy Research Working Paper, No. 8268. 
Bazen, S. And P. Moyes (2012): "Elitism and stochastic dominance," Social Choice and Welfare, 39, 207-251.

Behboodian, J., A. Dolati, and M. Úbeda-Flores (2007): "A multivariate version of Gini's rank association coefficient," Statistical Papers, 48, 295-304.

Bosmans, K., K. Decancq, and E. Ooghe (2015): "What do normative indices of multidimensional inequality really measure?" Journal of Public Economics, 130, 94-104.

Cowell, F. A. And E. Flachaire (2015): "Statistical Methods for Distributional Analysis," in Handbook on Income Distribution, volume 2, ed. by A. Atkinson and F. Bourguignon, vol. 2, 359-465.

Dalton, H. (1920): "The measurement of the inequality of incomes," The Economic Journal, 30, 25-49.

DECANCQ, K. (2012): "Elementary multivariate rearrangements and stochastic dominance on a Fréchet class," Journal of Economic Theory, 147, 1450 - 1459.

— (2014): "Copula-based measurement of dependence between dimensions of well-being," Oxford Economic Papers, 66, 681-701.

Decancq, K. and E. Schokkaert (2016): "Beyond GDP: Using equivalent incomes to measure well-being in Europe," Social Indicators Research, 126, $21-55$.

Dolati, A. And M. Úbeda-Flores (2006): "On measures of multivariate concordance," Journal of Probability and Statistical Science, 4, 147-163.

Epstein, L. G. And S. M. TAnny (1980): "Increasing generalized correlation: a definition and some economic consequences," The Canadian Journal of Economics / Revue canadienne d'Economique, 13, 16-34.

FERnÁndez-SÁnchez, J. AND M. ÚbedA-Flores (2018): "Constructions of copulas with given diagonal (and opposite diagonal) sections and some generalizations," Dependence Modeling, 6, 139-155.

García-Gómez, C., A. Pérez, and M. Prieto-Alaiz (2019): "Copulabased analysis of multivariate dependence patterns between dimensions of poverty in Europe," Mimeo. 
Genest, C., J. Nešlehová, And N. Ben Ghorbal (2010): "Spearman's footrule and Gini's gamma: a review with complements," Journal of Nonparametric Statistics, 22, 937-954.

Gini, C. (1914a): L'ammontare e la composizione della ricchezza delle nazioni, vol. 62, Fratelli Bocca.

(1914b): "Reprinted: On the measurement of concentration and variability of characters (2005)," Metron, 63, 3-38.

(1921): "Measurement of inequality of incomes," The Economic Journal, $31,124-126$.

Giorgi, G. M. (1990): "Bibliographic portrait of the Gini concentration ratio," Metron, 48, 183-221.

(1993): "A fresh look at the topical interest of the Gini concentration ratio," Metron, 51, 83-98.

Hamada, K. (1974): "Comment on Hadar and Russel (1974)," in Essays on Economic Behavior under Uncertainty, ed. by M. Balch, D. McFadden, and S. Wu, Amsterdam: North-Holland.

Joe, H. (1990): "Multivariate concordance," Journal of Multivariate Analysis, $35,12-30$.

(1997): Multivariate Models and Dependence Concepts. Monographs on Statistics and Applied Probability no. 73, London: Chapmann and Hall.

Kolm, S.-C. (1977): "Multidimensional egalitarianisms," The Quarterly Journal of Economics, 91, 1-13.

MaAsoumi, E. (1986): "The measurement and decomposition of multidimensional inequality," Econometrica, 54, 991-997.

Miller, D. (1995): "Complex equality," in Pluralism, Justice and Equality, ed. by D. Miller and M. Walzer, Oxford: Oxford University Press, 197-225.

MÜLler, A. (2013): "Duality Theory and Transfers for Stochastic Order Relations," in Stochastic Orders in Reliability and Risk, ed. by H. Li and X. Li, Springer New York, vol. 208 of Lecture Notes in Statistics, 41-57. 
Nelsen, R. B. (1991): "Copulas and association," in Advances in Probability Distributions with Given Marginals, ed. by G. Dall'Aglio, S. Kotz, and G. Salinetti, Dordrecht: Kluwer, 51-74.

(1996): "Nonparametric measures of multivariate association," in Distributions with Fixed Marginals and Related Topics, vol. 28 of IMS Lecture Notes, 223-232.

(2002): "Concordance and copulas: a survey," in Distributions with Given Marginals and Statistical Modelling, ed. by C. Cuadras, J. Fortiana, and J. M. R. Lallena, Dordrecht: Kluwer, 169-178.

(2006): Introduction to Copulas. Springer Series in Statistics, New York: Springer Verlag.

Nelsen, R. B. And M. Úbeda-Flores (2004): "The symmetric footrule is Gini's rank association coefficient," Communications in Statistics-Theory and Methods, 33, 195-196.

PÉrez, A. And M. Prieto (2015): "Measuring dependence between dimensions of poverty in Spain: An approach based on copulas," in 2015 Conference of the International Fuzzy Systems Association and the European Society for Fuzzy Logic and Technology (IFSA-EUSFLAT-15), Atlantis Press.

Pérez, A. And M. Prieto-Alaiz (2016): "Measuring the dependence among dimensions of welfare: A study based on Spearman's footrule and Gini's gamma," International Journal of Uncertainty, Fuzziness and KnowledgeBased Systems, 24, 87-105.

Quinn, C. (2007): "Using copulas to measure association between ordinal measures of health and income," Health, Econometrics and Data Group (HEDG) Working Papers 07/24.

- (2009): "Measuring income-related inequalities in health using a parametric dependence function," Health, Econometrics and Data Group (HEDG) Working Papers 09/24.

Salama, I. A. And D. Quade (2001): "The symmetric footrule," Communications in Statistics-Theory and Methods, 30, 1099-1109.

Sarabia, J. M. (2008): "A general definition of the Leimkuhler curve," Journal of Informetrics, 2, 156 - 163. 
Schmid, F. AND R. Schmidt (2007a): "Multivariate conditional versions of Spearman's rho and related measures of tail dependence," Journal of Multivariate Analysis, 98, 1123-1140.

(2007b): "Multivariate extensions of Spearman's rho and related statistics," Statistics 89 Probability Letters, 77, 407-416.

Sen, A. K. (1985): Commodities and Capabilities, Amsterdam and Oxford: North-Holland.

(1992): Inequality Reexamined, New York and Oxford: Russell Sage Foundation; Clarendon Press.

Sklar, A. (1959): "Fonctions de répartition à $n$ dimensions et leurs marges," Publications de l'Institut de Statistique de L'Université de Paris, 8, 229-231.

Spearman, C. (1904): "Reprinted: The proof and measurement of association between two things (2010)," International Journal of Epidemiology, 39, 11371150 .

TAYlor, M. (2007): "Multivariate measures of concordance," Annals of the Institute of Statistical Mathematics, 59, 789-806.

Tchen, A. (1980): "Inequalities for distributions with given marginals," The Annals of Probability, 8, 814-827.

Tkach, K. and C. Gigliarano (2018): "Multidimensional poverty measurement: dependence between well-being dimensions using copula function," RIEDS-Rivista Italiana di Economia, Demografia e Statistica-Italian Review of Economics, Demography and Statistics, 72, 89-100.

Trivedi, P. K. And D. M. Zimmer (2007): "Copula Modeling: An Introduction for Practitioners," Foundations and Trends in Econometrics, 1, 1-111.

Tsui, K. Y. (1995): "Multidimensional generalizations of the relative and absolute inequality indices: the Atkinson-Kolm-Sen approach," Journal of Economic Theory, 67, 251-265.

ÚBedA-Flores, M. (2005): "Multivariate versions of Blomqvist's beta and Spearman's footrule," Annals of the Institute of Statistical Mathematics, 57, $781-788$. 
Walzer, M. (1983): Spheres of Justice: A Defense of Pluralism and Equality, New York: Basic Books.

Weymark, J. A. (2006): "The normative approach to the measurement of multidimensional inequality," in Inequality and Economic Integration, ed. by F. Farina and E. Savaglio, London: Routledge. 
Copyright (c) 2020 @ the author(s). Discussion papers are in draft form. This discussion paper is distributed for purposes of comment and discussion only. It may not be reproduced without permission of the copyright holder. Copies of working papers are available from the author. 